\title{
Analysis of reporting return to work in studies comparing open with endoscopic carpal tunnel release: A review of randomized controlled trials
}

\author{
Olubimpe Ayeni MPH ${ }^{1}$, Achilleas Thoma MD MSc FRCSC FACS ${ }^{1,2}$, Ted Haines MD MSc FRCPC ${ }^{2,3}$, \\ Sheila Sprague MSc ${ }^{1}$
}

O Ayeni, A Thoma, T Haines, et al. Analysis of reporting return to work in studies comparing open with endoscopic carpal tunnel release: A review of randomly assigned controlled trials. Can J Plast Surg 2005;13(4):181-187.

BACKGROUND: In studies comparing open with endoscopic carpal tunnel release, return to work (RTW) is often cited as a primary outcome.

OBJECTIVE: The present study assessed the reporting of RTW and evaluated its usefulness in studies comparing these two methods of carpal tunnel release.

METHODS: A computerized search was conducted to find randomized controlled trials that compared open with endoscopic carpal tunnel release, with RTW as an outcome measure. The factors that were compared across the studies included definition of RTW, units quantifying RTW, measures of hand function, patients' type of employment, worker's compensation or insurance status, patients' handedness, unilateral or bilateral carpal tunnel release, and use of rehabilitation.

RESULTS: Fifteen studies met the inclusion criteria for the present systematic review. Of the 15 studies reviewed, there were seven definitions of RTW. All studies defined whether the patients underwent unilateral or bilateral carpal tunnel release but there was variability in the calculation of RTW when bilateral releases were performed. The impact of worker's compensation or insurance, type of work, handedness and rehabilitation were inconsistently addressed as factors affecting RTW.

CONCLUSIONS: Although RTW ideally reflects function and recovery, it is inadequately measured and reported. The present review revealed that, in studies comparing open carpal tunnel release with endoscopic carpal tunnel release, there is lack of uniformity in reporting RTW, which may contribute to the inconclusive results for RTW. Future research needs to ensure that RTW is used in a consistent manner.

Key Words: Carpal tunnel release; Outcome measures; Return to work; Systematic review

\section{Les déclarations de retour au travail dans des études comparant la libération ouverte à la libération endoscopique du tunnel carpien : Une analyse d'essais aléatoires et contrôlés}

HISTORIQUE : Dans les études comparant la libération ouverte à la libération endoscopique du tunnel carpien, le retour au travail (RAT) est souvent cité comme une issue primaire.

OBJECTIF : La présente étude vise à évaluer les déclarations de RAT et son utilité dans des études comparant ces deux méthodes de libération du tunnel carpien.

MÉTHODOLOGIE : Une recherche informatisée a été menée pour trouver des essais aléatoires et contrôlés comparant la libération ouverte à la libération endoscopique du tunnel carpien, le RAT constituant une mesure d'issue. Les facteurs qui étaient comparés dans les études sont la définition de RAT, les unités quantifiant le RAT, les mesures de la fonction de la main, le type d'emploi des patients, la rémunération des patients ou leur situation du point de vue de l'assurance, la prévalence manuelle des patients, la libération unilatérale ou bilatérale du tunnel carpien et le recours à la réadaptation.

RÉSULTATS : Quinze études respectaient les critères d'inclusion dans la présente analyse systématique. Elles comportaient sept définitions différentes du RAT. Toutes les études définissaient si les patients avaient subi une libération unilatérale ou bilatérale du tunnel carpien, mais on remarquait une variabilité dans le calcul de RAT en cas de libération bilatérale. Les répercussions de la rémunération ou de l'assurance des travailleurs, de leur type de travail, de leur prévalence manuelle et de leur réadaptation ne convergeaient pas toutes comme des facteurs influant sur le RAT.

CONCLUSIONS : Bien que le RAT reflète de manière idéale la fonction et le rétablissement, il est mesuré et déclaré de manière inadéquate. La présente analyse révèle que, dans des études comparant la libération ouverte à la libération endoscopique du tunnel carpien, la déclaration de RAT n'est pas uniforme, ce qui peut contribuer aux résultats non concluants quant au RAT. Dans le cadre de futures recherches, il faudrait s'assurer d'utiliser le RAT de manière uniforme.
$\mathrm{O}$ pen carpal tunnel release (OCTR) is the standard procedure for the surgical treatment of carpal tunnel syndrome. With the advent of minimally invasive surgery, endoscopic carpal tunnel release (ECTR) was introduced as an alternative to OCTR. Proponents of ECTR claimed that by dividing the transverse carpal ligament from within the carpal tunnel, structures above the ligament are left intact and this may hasten recovery and decrease postoperative morbidity (1). In addition, they posit that the smaller incision, which is made away from the middle of the palm, results in less pain and scarring (2). These benefits of ECTR are tempered by the cost of endoscopic equipment and training, the complexity of the surgery and the scarcity of studies about its long-term safety (3). Initial reports that compared OCTR with ECTR concluded that

\footnotetext{
${ }^{1}$ Department of Surgery, Division of Plastic and Reconstructive Surgery, St Josephs Healthcare, Surgical Outcomes Research Centre (SOURCE) and McMaster University; ${ }^{2}$ Department of Clinical Epidemiology and Biostatistics, McMaster University; ${ }^{3}$ Occupational Health Program, Health Sciences Centre, McMaster University, Hamilton, Ontario

Correspondence: Dr Achilleas Thoma, 206 James Street South, Suite 101, Hamilton, Ontario L8P 3A9. Telephone 905-523-0019, fax 905-523-0229,e-mail athoma@mcmaster.ca
} 
although ECTR has a shorter recovery period, it may also have a higher risk of complications (4).

A recent review (5) comparing the two methods of carpal tunnel release found no difference in terms of symptom relief, but there was conflicting evidence about hand function and return to work (RTW). Similarly, a meta-analysis (6) of studies that evaluated RTW in comparing the two techniques found a pooled odds ratio of 1.52 (95\% CI 0.28 to 8.34 ) favouring ECTR; the studies' results were statistically heterogeneous. These findings reflect the controversy over which method of carpal tunnel release offers better postoperative outcomes. They also raise the question of the degree to which differences or imprecision in the measurement of RTW could play a role in this inconclusiveness. Although both of these studies assessed RTW while comparing ECTR with OCTR, they focused on comparing the postoperative outcomes of the two techniques as opposed to addressing the factors that cause the heterogeneity in the reporting of RTW.

In studies investigating work-related upper extremity disorders, RTW is often cited as a primary outcome measure because it is helpful in assessing patient recovery and it provides information about the social and medical costs of surgery. On the contrary, RTW may be less helpful as a result of work- and nonwork-related confounding factors such as patient motivation, job availability, medicolegal issues and social concerns, which obscure the relationship between RTW and the surgical treatment (5). Despite these possible confounding factors, RTW is still a useful and easily calculated outcome measure.

When RTW is compared for different hand conditions in a study, the clinical and workplace factors that influence recovery can be difficult to distinguish. In choosing a single hand condition to study, carpal tunnel syndrome is ideal because its prevalence, clinical presentation and postoperative outcome are well established (7).

Given the controversy over the choice between ECTR and OCTR, valid results for RTW could have an impact on the choice of procedure when assessing their risks and benefits. The aim of the present study was to assess the quality and usefulness of reporting of RTW in published randomized controlled trials comparing OCTR with ECTR.

\section{METHODS}

To identify eligible studies, a computerized search was conducted in the electronic database MEDLINE (January 1966 to January 2005) and in the Cochrane Central Register of Controlled Trials (updated for the fourth quarter of 2004) for the period from 1990 to 2004, inclusive. The search was limited to this period because the first reports of ECTR were published in 1989 (8). The following search terms and boolean operators were combined: "open OR endoscopic" AND "carpal tunnel release OR carpal tunnel surgery". The bibliography of each article that met the inclusion criteria (described below) was reviewed to find additional eligible studies. Translated versions of studies published in French, Dutch, German and Portuguese were obtained.

To be included, a study had to meet the following criteria: the study had to be designed as a randomized controlled trial; the study had to compare OCTR with ECTR; and RTW had to be an outcome measure. Two investigators (OA and SS) independently reviewed the citations for relevance and carried out data abstraction. Consensus was used to resolve disagreements.

For each study, time to RTW following ECTR and OCTR was recorded. The following data from eligible studies were then abstracted: definition of RTW, units used to quantify RTW, measures of hand function, patients' type of employment, worker's compensation or insurance status, patients' handedness, unilateral or bilateral carpal tunnel release and use of rehabilitation.

\section{RESULTS}

Fifteen studies met the inclusion criteria set for the present study (8-22) and the studies' features are summarized in Tables 1 to 3. Of the ineligible studies, 11 were excluded because they were not randomized (23-33), four because they did not compare ECTR with OCTR (34-37) and two because RTW was not an outcome measure (38-39).

Of the 15 included studies, there were seven different definitions of RTW. These definitions were: time to RTW $(9,11,17,20,22)$; sick leave (16); absence from work (8); time to RTW or activities of daily living (13); time off from work $(14,15,18)$; inability to work $(10,19,21)$; and per cent of patients returning to work at two weeks, one month and three months (12).

RTW was quantified using three different units. Twelve studies $(9-11,13-15,16-18,20-22)$ used days and two $(8,19)$ used weeks as the unit of time. One study (12) calculated the per cent of patients returning to work at two weeks, one month and three months, thereby quantifying RTW as a percentage of patients and using a combination of weeks and months.

Hand function was assessed across the studies using similar measures. In 14 of 15 studies (8-15,17-22), grip and/or pinch strength were measured. With regard to hand function, 10 of 14 studies $(8-13,17,20-22)$ found that patients in the ECTR group recovered grip and/or pinch strength sooner than those in the OCTR group. Three studies $(14,18,19)$ found no significant differences in hand function between the two groups and one study (15) found an improvement in strength in one of its OCTR groups. In addition, in seven of 10 studies $(9-11,13,20$ 22) where there was faster recovery of grip and/or pinch strength in the ECTR group, there was also faster RTW for the ECTR group. In the remaining three studies with faster recovery of grip and/or pinch strength in the ECTR group, there was no difference in RTW between the two groups $(8,12,17)$.

Patient occupation was defined in 11 of 15 studies (10$14,16,18-22)$. In four of these studies $(8,10,12,18)$, associations were made between type of work and ability to RTW with all four finding no significant differences in RTW based on occupation. None of the studies provided ergonomic information about the jobs such as hand forces, postures, paces or task duration.

Worker's compensation was addressed in five studies. In one study, none of the enrolled patients received compensation (16). In two studies, no comparison was made about RTW in patients receiving and not receiving compensation $(11,22)$. Finally, one study (9) found that RTW was slower for patients receiving compensation, while the last study found no difference (17). In the studies reviewed, there was no documentation of insurance or benefits status other than worker's compensation.

Ten studies $(10,11,13-15,16,18,20-22)$ stated the number of dominant hand operations that were performed and if the ECTR and OCTR groups were comparable in terms of the number of patients having operations on their dominant hand. Although most studies listed this factor as a patient characteristic, none of the studies discussed how having surgery on the dominant or nondominant hand affected RTW. 
TABLE 1

Characteristics of studies comparing endoscopic carpal tunnel release (ECTR) and open carpal tunnel release (OCTR)

\begin{tabular}{|c|c|c|c|c|c|c|c|}
\hline First author & $\begin{array}{l}\text { Patients/ } \\
\text { hands }(n)\end{array}$ & $\begin{array}{l}\text { ECTR \& } \\
\operatorname{OCTR}(n)\end{array}$ & $\begin{array}{l}\text { ECTR } \\
\text { technique }\end{array}$ & $\begin{array}{l}\text { OCTR } \\
\text { technique }\end{array}$ & $\begin{array}{l}\text { Unilateral or } \\
\text { bilateral releases } \\
\text { or both }\end{array}$ & Primary and secondary outcomes & $\begin{array}{l}\text { Overall difference in } \\
\text { RTW between ECTR } \\
\text { and OCTR }\end{array}$ \\
\hline Agee (9) & $122 / 147$ & $\begin{array}{l}82 \text { ECTR } \\
65 \text { OCTR }\end{array}$ & $\begin{array}{l}\text { One-portal } \\
\text { Agee }\end{array}$ & $\begin{array}{l}\text { Classic } \\
\text { incision }\end{array}$ & Both & $\begin{array}{l}\text { RTW, complications, employment status, return } \\
\text { of hand use for ADL, postoperative symptoms, } \\
\text { clinical examination (grip and pinch strength, } \\
\text { monofilament sensory exam, motor testing) }\end{array}$ & $\begin{array}{l}\text { Faster RTW for ECTR } \\
\text { ( } 25 \text { days vs } 46.5 \text { days) } \\
\mathrm{P}<0.01\end{array}$ \\
\hline Benedetti (10) & $45 / 45$ & $\begin{array}{l}22 \text { OCTR } \\
23 \text { ECTR }\end{array}$ & $\begin{array}{l}\text { One-portal } \\
\text { Agee }\end{array}$ & $\begin{array}{l}\text { Longitudinal } \\
\text { incision }\end{array}$ & Unilateral & $\begin{array}{l}\text { RTW, grip and pinch strength, complications, } \\
\text { fitness of hand, symptom relief }\end{array}$ & $\begin{array}{l}\text { Faster RTW for ECTR } \\
\text { ( } 24.5 \text { days vs } 41.9 \text { days) } \\
P=0.003\end{array}$ \\
\hline Brown (11) & $145 / 169$ & $\begin{array}{l}84 \text { ECTR } \\
85 \text { OCTR }\end{array}$ & $\begin{array}{l}\text { Two-portal } \\
\text { extrabursal } \\
\text { Chow }\end{array}$ & $\begin{array}{l}\text { Classic } \\
\text { incision }\end{array}$ & Both & $\begin{array}{l}\text { Relief of symptoms, patient satisfaction. Secondary } \\
\text { outcomes: interstitial carpal pressure, grip and } \\
\text { pinch strength, two-point discrimination, scar and } \\
\text { pillar, tenderness, monofilament testing, ADL, } \\
\text { RTW, time and cost, complications }\end{array}$ & $\begin{array}{l}\text { Faster RTW for ECTR } \\
\text { (14 days vs } 28 \text { days) } \\
P<0.05\end{array}$ \\
\hline Dumontier (12) & $96 / 96$ & $\begin{array}{l}40 \text { OCTR } \\
56 \text { ECTR }\end{array}$ & $\begin{array}{l}\text { Two-portal } \\
\text { extrabursal } \\
\text { Chow }\end{array}$ & $\begin{array}{l}\text { Short } \\
\text { incision }\end{array}$ & Unilateral & $\begin{array}{l}\text { RTW, numbness, pain, grip strength, finger } \\
\text { mobility, complications }\end{array}$ & $\begin{array}{l}\text { RTW was sooner in } \\
\text { OCTR group, but } \\
\text { difference was not } \\
\text { significant }(P=0.13)\end{array}$ \\
\hline Erdmann (13) & $71 / 105$ & $\begin{array}{l}53 \text { ECTR } \\
52 \text { OCTR }\end{array}$ & $\begin{array}{l}\text { Two-portal } \\
\text { extrabursal } \\
\text { Chow }\end{array}$ & $\begin{array}{l}\text { Short } \\
\text { incision }\end{array}$ & Both & $\begin{array}{l}\text { Time to RTW or ADL, grip and pinch } \\
\text { strength, complication rate, carpal tunnel pain, } \\
\text { nerve conduction }\end{array}$ & $\begin{array}{l}\text { Faster RTW for ECTR } \\
\text { (14 days vs } 39 \text { days) } \\
\mathrm{P}<0.005\end{array}$ \\
\hline Ferdinand (14) & $25 / 50$ & $\begin{array}{l}25 \text { ECTR } \\
25 \text { OCTR }\end{array}$ & $\begin{array}{l}\text { One-portal } \\
\text { Agee }\end{array}$ & $\begin{array}{l}\text { Classic } \\
\text { incision }\end{array}$ & Bilateral & $\begin{array}{l}\text { Time off work, operating times, return of muscle } \\
\text { strength, hand function, grip strength, manual } \\
\text { dexterity, sensation }\end{array}$ & No difference \\
\hline Foucher (15) & $249 / 251$ & $\begin{array}{l}54 \text { ECTR } \\
69 \text { OCTR } \\
59 \text { AL1 } \\
69 \text { AL2 }\end{array}$ & $\begin{array}{l}\text { One-portal } \\
\text { Agee }\end{array}$ & $\begin{array}{l}\text { Classic } \\
\text { incision }\end{array}$ & Both & Time off work, postoperative strength, palmar pain & No difference \\
\hline Hoefnagels (8) & $176 / 176$ & $\begin{array}{l}85 \text { ECTR } \\
91 \text { OCTR }\end{array}$ & $\begin{array}{l}\text { One-portal } \\
\text { Agee }\end{array}$ & $\begin{array}{l}\text { Classic } \\
\text { incision }\end{array}$ & Unilateral & $\begin{array}{l}\text { Absence from work, palmar pain, complications, } \\
\text { patient satisfaction, cost }\end{array}$ & $\begin{array}{l}\text { No difference; } \\
38 \text { patients absent from } \\
\text { work after ECTR vs } \\
41 \text { patients absent } \\
\text { from work after OCTR }\end{array}$ \\
\hline Jacobsen (16) & $29 / 32$ & $\begin{array}{l}16 \text { ECTR } \\
16 \text { OCTR }\end{array}$ & $\begin{array}{l}\text { Two-portal } \\
\text { transbursal } \\
\text { Chow }\end{array}$ & $\begin{array}{l}\text { Longitudinal } \\
\text { incision }\end{array}$ & Both & $\begin{array}{l}\text { Sick leave, symptom relief, total number of } \\
\text { analgesics, two-point discrimination }\end{array}$ & $\begin{array}{l}\text { No significant difference } \\
\text { (ECTR mean sick leave } \\
17 \text { days, OCTR mean } \\
\text { sick leave } 19 \text { days) }\end{array}$ \\
\hline MacDermid (17) & $\begin{array}{l}123 / \\
\text { Unknown }\end{array}$ & $\begin{array}{l}91 \text { ECTR } \\
32 \text { OCTR }\end{array}$ & $\begin{array}{l}\text { Two-portal } \\
\text { Chow }\end{array}$ & $\begin{array}{l}\text { Standard } \\
\text { long } \\
\text { incision }\end{array}$ & $\begin{array}{l}\text { Not } \\
\text { mentioned }\end{array}$ & $\begin{array}{l}\text { Symptom severity, nerve/vascular complications. } \\
\text { Secondary outcomes: RTW, McGill pain } \\
\text { questionnaire, grip strength, pinch strength, } \\
\text { sensory threshold }\end{array}$ & No significant difference \\
\hline Saw (18) & $150 / 150$ & $\begin{array}{l}74 \text { ECTR } \\
76 \text { OCTR }\end{array}$ & $\begin{array}{l}\text { One-portal } \\
\text { Agee }\end{array}$ & $\begin{array}{l}\text { Standard } \\
2 \mathrm{~cm} \\
\text { incision }\end{array}$ & Unilateral & $\begin{array}{l}\text { Sick leave. Secondary outcomes: operation time, } \\
\text { carpal tenderness, grip strength, Levine } \\
\text { symptoms severity scale, cost-effectiveness } \\
\text { analysis, complications }\end{array}$ & $\begin{array}{l}\text { Faster RTW for ECTR } \\
\text { (18 days vs } 26 \text { days off } \\
\text { work) } \mathrm{P}=0.005\end{array}$ \\
\hline Schafer (19) & $101 / 101$ & $\begin{array}{l}54 \text { OCTR } \\
47 \text { ECTR }\end{array}$ & $\begin{array}{l}\text { One-portal } \\
\text { Agee }\end{array}$ & $\begin{array}{l}\text { Short } \\
\text { incision }\end{array}$ & Unilateral & $\begin{array}{l}\text { Inability to work, pain, thenar atrophy, grip and } \\
\text { pinch strength, distal motor latency, two-point } \\
\text { discrimination }\end{array}$ & $\begin{array}{l}\text { Faster RTW for ECTR } \\
\text { (inability to work } 3.9 \\
\text { days vs } 5.3 \text { weeks) }\end{array}$ \\
\hline Sennwald (20) & $47 / 47$ & $\begin{array}{l}25 \text { ECTR } \\
22 \text { OCTR }\end{array}$ & $\begin{array}{l}\text { One-portal } \\
\text { Agee }\end{array}$ & $\begin{array}{l}\text { Longitudinal } \\
\text { incision }\end{array}$ & Unilateral & $\begin{array}{l}\text { Time out of work, pain, grip and key-strength, } \\
\text { complications }\end{array}$ & $\begin{array}{l}\text { Faster RTW for ECTR } \\
\text { (time out of work } \\
\text { significantly reduced } \\
\text { after ECTR compared } \\
\text { with OCTR) } \mathrm{P}=0.0000\end{array}$ \\
\hline Stark (21) & $20 / 40$ & $\begin{array}{l}20 \text { ECTR } \\
20 \text { OCTR }\end{array}$ & $\begin{array}{l}\text { One-portal } \\
\text { Agee }\end{array}$ & $\begin{array}{l}\text { Classic } \\
\text { incision }\end{array}$ & Bilateral & $\begin{array}{l}\text { Inability to work, pain, grip and key-strength, } \\
\text { complications, two-point discrimination }\end{array}$ & $\begin{array}{l}\text { Faster RTW for ECTR } \\
\text { (inability to work lasted } \\
20 \text { days, ECTR; } 30 \\
\text { days, OCTR) } P<0.001\end{array}$ \\
\hline Trumble (22) & $147 / 192$ & $\begin{array}{l}97 \text { ECTR } \\
95 \text { OCTR }\end{array}$ & $\begin{array}{l}\text { One-portal } \\
\text { Agee }\end{array}$ & $\begin{array}{l}\text { Longitudinal } \\
\text { incision }\end{array}$ & Both & $\begin{array}{l}\text { Time to RTW, pinch and grip strength, } \\
\text { Jebsen-Taylor hand function test, carpal tunnel } \\
\text { syndrome functional status score, carpal tunnel } \\
\text { syndrome symptom severity score, satisfaction. } \\
\text { Secondary outcome: complications }\end{array}$ & $\begin{array}{l}\text { Faster RTW for ECTR } \\
\text { (median time to work } \\
18 \text { days ECTR and } \\
38 \text { days OCTR) } \\
P=0.0086\end{array}$ \\
\hline
\end{tabular}

*Primary outcomes are listed for all studies and secondary outcomes are listed only when indicated; †With anterior ligamentoplasty. ADL Activities of daily living; AL Anterior ligamentoplasty types 1 and 2; RTW Return to work; vs Versus 
TABLE 2

Definitions of return to work (RTW), hand function, hand dominance and worker's compensation in studies comparing endoscopic carpel tunnel release (ECTR) and open carpal tunnel release (OCTR)

\begin{tabular}{|c|c|c|c|c|c|c|}
\hline First author & Definition of RTW & Units & Hand function & Type of work & Hand dominance & Worker's compensation \\
\hline Agee (9) & Time for RTW & Days & Pinch and grip strength & Not mentioned & Not mentioned & $\begin{array}{l}\text { Patients receiving compensation } \\
\text { RTW later ( } 71 \text { days vs } 78 \text { days) }\end{array}$ \\
\hline Benedetti (10) & Inability to work & Days & Pinch grip, grip strength & Light and heavy work & Mentioned & Not mentioned \\
\hline Brown (11) & $\begin{array}{l}\text { Interval until patient } \\
\text { can RTW }\end{array}$ & Days & $\begin{array}{l}\text { Grip strength and key } \\
\text { pinch strength }\end{array}$ & $\begin{array}{l}\text { Work at home, retired, work } \\
\text { outside home (professional, } \\
\text { managerial, manual, clerical/ } \\
\text { technical, business) }\end{array}$ & Mentioned & $\begin{array}{l}\text { Mentioned. No association made } \\
\text { to RTW }\end{array}$ \\
\hline Dumontier (12) & $\begin{array}{l}\text { Time to RTW; \% pts } \\
\text { returning to work at } \\
2 \text { weeks, } 1 \text { month } \\
\text { and } 3 \text { months }\end{array}$ & $\%$ pts* & Grip strength & $\begin{array}{l}\text { Manual vs clerical/retired/ } \\
\text { unemployed }\end{array}$ & Not mentioned & Not mentioned \\
\hline Erdmann (13) & $\begin{array}{l}\text { Time to RTW } \\
\text { or ADL }\end{array}$ & Days & Pinch and grip strength & $\begin{array}{l}\text { Carpentry, weightlifting, typing, } \\
\text { refuse collecting, bricklaying }\end{array}$ & Mentioned & Not mentioned \\
\hline Ferdinand (14) & Time out of work & Days & $\begin{array}{l}\text { Grip strength, Jebsen } \\
\text { hand function test }\end{array}$ & $\begin{array}{l}\text { Retired, employed (specific job } \\
\text { listed for all nonretired pts) }\end{array}$ & Not mentioned & Not mentioned \\
\hline Foucher (15) & Time out of work & Days & Grip strength & Not mentioned & Mentioned & Not mentioned \\
\hline Hoefnagels (8) & Absence from work & Weeks & Pinch and grip strength & Not mentioned & Not mentioned & Not mentioned \\
\hline Jacobsen (16) & Sick leave & Days & $\begin{array}{l}\text { No measures of pinch } \\
\text { or grip strength; symptom } \\
\text { relief measured }\end{array}$ & Employed & Mentioned & $\begin{array}{l}\text { No patients received } \\
\text { compensation }\end{array}$ \\
\hline MacDermid (17) & $\begin{array}{l}\text { Time that those who } \\
\text { were employed took } \\
\text { to RTW }\end{array}$ & Days & $\begin{array}{l}\text { Pinch and grip strength, } \\
\text { symptom severity scale }\end{array}$ & Not mentioned & Not mentioned & $\begin{array}{l}\text { Mentioned. No association } \\
\text { made to RTW }\end{array}$ \\
\hline Saw (18) & $\begin{array}{l}\text { Number of days off } \\
\text { work }\end{array}$ & Days & $\begin{array}{l}\text { Grip strength, Levine's } \\
\text { symptom severity and } \\
\text { functional status scales }\end{array}$ & $\begin{array}{l}\text { Employed vs self-employed; } \\
\text { manual vs nonmanual work }\end{array}$ & Mentioned & Not mentioned \\
\hline Schafer (19) & Inability to work & Weeks & Grip strength & Occupation recorded & Not mentioned & Not mentioned \\
\hline Sennwald (20) & Time out of work & Days & $\begin{array}{l}\text { Grip and key pinch } \\
\text { strength }\end{array}$ & $\begin{array}{l}\text { Patients had similar occupation } \\
\text { status }\end{array}$ & Mentioned & Not mentioned \\
\hline Stark (21) & Inability to work & Days & Grip strength & $\begin{array}{l}\text { Light vs heavy work; use of } \\
\text { vibrating tools at work }\end{array}$ & Mentioned & Not mentioned \\
\hline Trumble (22) & Time until RTW & Days & $\begin{array}{l}\text { Pinch and grip strength, } \\
\text { Jebsen-Taylor hand } \\
\text { function test, carpal tunnel } \\
\text { syndrome functional status } \\
\text { score, carpal tunnel } \\
\text { syndrome symptom } \\
\text { severity score }\end{array}$ & $\begin{array}{l}\text { Work at home, work outside, } \\
\text { retired }\end{array}$ & Mentioned & $\begin{array}{l}\text { Mentioned. No association made } \\
\text { to RTW }\end{array}$ \\
\hline
\end{tabular}

${ }^{*} R T W$ at 2 weeks, 1 month and 3 months. ADL Activities of daily living; pts Patients; vs Vs

There were eight studies that involved bilateral release in which there were three scenarios. In four studies $(9,16,21,22)$, patients had surgeries on two different days, in two studies $(13,14)$ the patients had the surgeries on the same day and in one study (11) there were both same day and different day operations. The timing of the operations was unclear in another study involving bilateral releases (15).

With respect to the calculation of RTW in the studies involving bilateral releases, two $(14,21)$ of the studies involved only bilateral releases. In addition, one study (9) excluded the patients that underwent bilateral releases from the calculation of RTW, two studies $(11,22)$ rated RTW separately after each procedure and in four studies $(14,15,16,21)$, it was unclear how RTW was computed for the bilateral releases. Finally, postoperative hand therapy was mentioned in two of 15 studies $(21,22)$.

\section{DISCUSSION}

The present systematic review included 15 randomized controlled trials comparing OCTR with ECTR. Although the nine factors that were assessed in the present study may have an impact on RTW, the present review reveals that they have been defined or addressed inconsistently. This variability raises concerns about the reliability of the reporting of RTW, which in turn has an impact on making a treatment choice between ECTR and OCTR. The seven different definitions of RTW that were revealed in the present review exemplify the lack of uniformity in conceptualizing it. While some definitions were similar ('time off from work' and 'inability to work'), others differed widely ('sick leave,' 'time to RTW,' 'time to RTW or activities of daily living' and 'per cent of patients returning to work at two weeks, one month and three months'). In addition to the varying definitions of RTW, days, weeks and months were used to quantify time in the different studies. Imprecision would result from combining these to a single unit.

Despite the universal reporting of grip and pinch strength, there was inconsistent use of hand function tests with some studies using tools such as the Jebsen hand function test $(14,22)$, the Levine symptom severity and functional status 
TABLE 3

Factors affecting return to work (RTW) in studies comparing endoscopic carpal tunnel release (ECTR) and open carpal tunnel release (OCTR)

\begin{tabular}{|c|c|c|c|c|c|}
\hline First author & $\begin{array}{l}\text { Impact of hand } \\
\text { function on RTW }\end{array}$ & $\begin{array}{l}\text { Impact of type } \\
\text { of work on RTW }\end{array}$ & $\begin{array}{l}\text { Impact of hand } \\
\text { dominance on RTW }\end{array}$ & $\begin{array}{l}\text { Impact of rehabilitation } \\
\text { on RTW }\end{array}$ & $\begin{array}{l}\text { Impact of worker's } \\
\text { compensation }\end{array}$ \\
\hline Agee (9) & $\begin{array}{l}\text { ECTR group returned to } \\
\text { preoperative or greater } \\
\text { strength more quickly } \\
\text { than OCTR group }\end{array}$ & $\begin{array}{l}\text { Patients had 'similar } \\
\text { employment status' }\end{array}$ & Not mentioned & Not mentioned & $\begin{array}{l}\text { Patients receiving compensation } \\
\text { RTW later ( } 71 \text { days vs } 78 \text { days) }\end{array}$ \\
\hline Benedetti (10) & $\begin{array}{l}\text { ECTR group regained } \\
\text { strength faster post- } \\
\text { operation and at } 3 \text { months }\end{array}$ & $\begin{array}{l}\text { No difference between light } \\
\text { and heavy work }\end{array}$ & $\begin{array}{l}\text { Mentioned; not linked back } \\
\text { to RTW }\end{array}$ & Not mentioned & Not mentioned \\
\hline Brown (11) & $\begin{array}{l}\text { ECTR group had better } \\
\text { pinch strength }\end{array}$ & $\begin{array}{l}\text { Mentioned; not linked to } \\
\text { RTW }\end{array}$ & $\begin{array}{l}\text { Mentioned; not linked back } \\
\text { to RTW }\end{array}$ & Not mentioned & $\begin{array}{l}\text { Mentioned; no association made } \\
\text { to RTW }\end{array}$ \\
\hline Dumontier (12) & $\begin{array}{l}\text { Better grip strength } \\
\text { recovery at } 1 \text { month and } \\
3 \text { months for ECTR group }\end{array}$ & $\begin{array}{l}\text { Grip strength recovery faster } \\
\text { for ECTR group at } 3 \text { months } \\
\text { for light and heavy manual } \\
\text { workers }\end{array}$ & $\begin{array}{l}\text { Mentioned; not linked back } \\
\text { to RTW }\end{array}$ & Not mentioned & Not mentioned \\
\hline Erdmann (13) & $\begin{array}{l}\text { Improvement in grip and } \\
\text { pinch strength for ECTR } \\
\text { group in postoperation period }\end{array}$ & Diverse & $\begin{array}{l}\text { Mentioned; not linked back } \\
\text { to RTW }\end{array}$ & Not mentioned & Not mentioned \\
\hline Ferdinand (14) & $\begin{array}{l}\text { No significant differences } \\
\text { in Jebsen hand function } \\
\text { test or grip strength } \\
\text { between ECTR and } \\
\text { OCTR groups }\end{array}$ & $\begin{array}{l}\text { Occupations listed; } \\
\text { bilateral }\end{array}$ & $\begin{array}{l}\text { Mentioned; not linked back } \\
\text { to RTW }\end{array}$ & Not mentioned & Not mentioned \\
\hline Foucher (15) & $\begin{array}{l}\text { Strength improved in the } \\
\text { anterior ligamentoplasty } \\
\text { group }\end{array}$ & Not mentioned & $\begin{array}{l}\text { Mentioned; not linked back } \\
\text { to RTW }\end{array}$ & Not mentioned & Not mentioned \\
\hline Hoefnagels (8) & $\begin{array}{l}\text { Better grip strength for } \\
\text { ECTR group at } 3 \text { months }\end{array}$ & $\begin{array}{l}\text { No difference in RTW } \\
\text { between light and heavy } \\
\text { work }\end{array}$ & Not mentioned & Not mentioned & Not mentioned \\
\hline Jacobsen (16) & $\begin{array}{l}\text { No measures of pinch or } \\
\text { grip strength }\end{array}$ & $\begin{array}{l}\text { Employed; no association } \\
\text { to RTW }\end{array}$ & $\begin{array}{l}\text { Mentioned; not linked back } \\
\text { to RTW }\end{array}$ & Not mentioned & $\begin{array}{l}\text { No patients received } \\
\text { compensation }\end{array}$ \\
\hline MacDermid (17) & $\begin{array}{l}\text { Better grip strength at } 1 \text { and } \\
6 \text { weeks in ECTR group }\end{array}$ & No association to RTW & Not addressed & Not mentioned & $\begin{array}{l}\text { Mentioned; no association made } \\
\text { to RTW }\end{array}$ \\
\hline Saw (18) & No difference & $\begin{array}{l}\text { Adjusting for manual and } \\
\text { nonmanual workers made } \\
\text { no difference in RTW }\end{array}$ & $\begin{array}{l}\text { Mentioned; not linked back } \\
\text { to RTW }\end{array}$ & Not mentioned & Not mentioned \\
\hline Schafer (19) & $\begin{array}{l}\text { Similar recovery of strength } \\
\text { after } 4 \text { to } 12 \text { weeks }\end{array}$ & Occupation recorded & Not mentioned & Not mentioned & Not mentioned \\
\hline Sennwald (20) & $\begin{array}{l}\text { Better grip strength at } \\
12 \text { weeks for ECTR } \\
\text { group }\end{array}$ & $\begin{array}{l}\text { Patients had similar } \\
\text { occupation status; no } \\
\text { association made to RTW }\end{array}$ & Mentioned & Not mentioned & Not mentioned \\
\hline Stark (21) & $\begin{array}{l}\text { Better strength in ECTR } \\
\text { group at } 2 \text { and } 4 \text { weeks }\end{array}$ & Light or heavy work & Mentioned & Offered to patients & Not mentioned \\
\hline Trumble (22) & $\begin{array}{l}\text { Faster recovery of grip and } \\
\text { pinch strength for ECTR }\end{array}$ & $\begin{array}{l}\text { Work at home, work outside, } \\
\text { retired }\end{array}$ & Mentioned & Offered to patients & $\begin{array}{l}\text { Mentioned; no association } \\
\text { made to RTW }\end{array}$ \\
\hline
\end{tabular}

vs Versus

scales $(8,18)$ and the carpal tunnel release questionnaire $(22)$. The use of these various tools to assess hand function results in difficulty when comparing the studies.

Occupational characteristics are an important consideration because it is important to know the type of job the patient had both before and after surgery (40). Some employers offer employees the chance to return to modified work, with a smaller workload or different duties, after surgery. In addition, some employers encourage their employees to RTW the day after their surgery and the employees undertake nonstrenuous work duties with the normal hand. In this way, the employer may incur lower compensation or sick benefit costs. The ergonomic characteristics of work - hand and wrist forces, paces, postures and durations - may have played a role in the causation of the patient's carpal tunnel syndrome and are likely to affect recovery $(41,42)$. The nature of the workplace was unclear in nine of the studies that were reviewed $(8,9,15-20,22)$, and it was unclear if the patient was returning to the same or different duties in all of the studies reviewed.

There is evidence that among patients receiving compensation, RTW is slower among those not receiving compensation $(17,43)$. This is most likely because there is less financial incentive to RTW more quickly if the employee's income is being replaced. With respect to compensation status, 11 studies were conducted in Europe (8,10,12-16,18-21), three were conducted in North America $(9,17,22)$ and one was a collaborative effort between European and North American researchers (11). The country where the studies were conducted 
may have an impact on compensation as a result of international variations in compensation but this is difficult to conclude if compensation status is not recorded.

It is anticipated that there would be a difference in recovery, or RTW, if surgery was performed on the patient's dominant hand as opposed to the nondominant hand. This factor was assessed because it has an impact on how balanced the study groups are and if it is not addressed, it could introduce a selection bias, with regard to RTW as an outcome. Since carpal tunnel syndrome may affect one or both hands, it is also important to note if patients have had bilateral or unilateral surgery.

Having both hands operated has obvious implications for RTW. For the patients that underwent unilateral release, the calculation of RTW was simple, but it was more challenging to determine the impact of bilateral release when the releases did not occur closely in time (for instance, within a few days of each other). In the six studies that involved both bilateral and unilateral releases, RTW was not compared between the patients undergoing bilateral and unilateral releases $(9,11,13,15,16,22)$.

Flaws in study design were revealed in some of the studies involving bilateral releases. In two studies, patients underwent bilateral releases on the same day with one hand randomly assigned to ECTR and the other to $\operatorname{OCTR}(13,14)$. As a result, it is difficult to assess how each procedure contributed to RTW and the first surgery may have influenced the outcome of the second. In another study, two patients crossed over from the ECTR group to the OCTR group (8). Since these patients were excluded from the calculation of RTW, an intention to treat analysis was not used. Finally, in one study, some patients did not receive the procedure that they were randomly assigned to receive because they refused to undergo OCTR after having ECTR on the first hand (9). This can destroy the prognostic balance of random assignment, thereby introducing bias into the study.

Rehabilitation or hand therapy after surgery is beneficial (44) yet this factor was mentioned in only two studies $(21,22)$. In those two studies, the details about the amount and type of

\section{REFERENCES}

1. Gerritsen AA, Uitdehaag BM, van Geldere D, Scholten RJ, de Vet HC, Bouter LM. Systematic review of randomized clinical trials of surgical treatment for carpal tunnel syndrome. Br J Surg 2001;88:1285-95.

2. Scholten RJ, Gerritsen AM, Uitdehaag BM, van Geldere D, de Vet HC, Bouter LM. Surgical treatment options for carpal tunnel syndrome. Cochrane Database of Syst Rev 2005;(3).

3. Deune EG, Mackinnon SE. Endoscopic carpal tunnel release. The voice of polite dissent. Clin Plast Surg 1996;23:487-505.

4. Boeckstyns ME, Sorensen AI. Does endoscopic carpal tunnel release have a higher rate of complications than open carpal tunnel release? An analysis of published series. J Hand Surg [Br] 1999;24:9-15.

5. Thoma A, Veltri K, Haines T, Duku, E. A systematic review of reviews comparing the effectiveness of endoscopic and open carpal tunnel decompression. Plast Reconstr Surg 2004;113:1184-91.

6. Thoma A, Veltri K, Haines T, Duku E. A meta-analysis of randomized controlled trials comparing endoscopic and open carpal tunnel decompression. Plast Reconstr Surg 2004;114:1137-46.

7. Katz JN, Amick BC 3rd, Keller R, et al. Determinants of work absence following surgery for carpal tunnel syndrome. Am J Ind Med 2005; 47:120-30.

8. Hoefnagels WA, van Kleef JG, Mastenbroek GG, de Blok JA, Breukelman AJ, de Krom MC. [Surgical treatment of carpal tunnel syndrome: Endoscopic or classical (open)? A prospective randomized trial.] Ned Tijdschr Geneeskd 1997;141:878-82.

9. Agee JM, McCarroll HR Jr, Tortosa RD, Berry DA, Szabo RM, Peimer CA. Endoscopic release of the carpal tunnel: A randomized prospective multicenter study. J Hand Surg [Am] 1992;17:987-95. therapy were not stated and it was unclear if the therapy had an impact on the patients' ability to RTW. It is important to report such information because therapy after hand surgery could potentially affect patient recovery as well as RTW.

Because there is conflicting evidence about the postoperative benefits of ECTR and OCTR, RTW would, ideally, be a useful aid in choosing a technique, but its usefulness depends on how it is defined and reported. To refine the definition of RTW in future research, certain specific changes can be made. We would recommend, as a definition of RTW, the time to RTW, calculated from the day of operation until the first day back to work. The most precise unit to use is days. Hand function can be better defined by using the same questionnaires or tests to assess hand function and tests of hand function should be separated from activities of daily living.

It is important to record type of work and how job characteristics, including ergonomical variables, impact on RTW rates. Worker's compensation, or insurance status, and rehabilitation are other factors worth noting. Interpretation of RTW can also be improved by ensuring that there are a comparable number of dominant hand operations in the groups being compared. The impact of bilateral release can be compared with unilateral release, if the procedures are conducted within a few days of each other.

Future research can examine other factors that affect RTW such as employers' wishes or pressure, insurance carriers and the role that company doctors or RTW programs play in employee recovery. Geographic variations in RTW, additional hand surgeries at time of carpal tunnel release, and variations in technique could also offer insight into trends that occur in RTW.

RTW is challenging to interpret and the present review revealed the heterogeneity that exists in the reporting of RTW in addition to the inconsistent reporting of patient and physician factors that may affect RTW. This outcome measure can be further refined to ensure that it can be a valuable, standardized tool for assessing patient recovery after carpal tunnel release.

10. Benedetti VR, Sennwald G. [Agee endoscopic decompression of the median nerve: Prospective study with comparison to open decompression.] Handchir Mikrochir Plast Chir 1996;28:151-5.

11. Brown RA, Gelberman RH, Seiler JG 3rd, et al. Carpal tunnel release. A prospective, randomized assessment of open and endoscopic methods. J Bone Joint Surg Am 1993;75:1265-75.

12. Dumontier C, Sokolow C, Leclercq C, Chauvin P. Early results of conventional versus two-portal endoscopic carpal tunnel release. A prospective study. J Hand Surg [Br] 1995;20:658-62.

13. Erdmann MW. Endoscopic carpal tunnel decompression. J Hand Surg [Br] 1994;19:5-13.

14. Ferdinand RD, MacLean JG. Endoscopic versus open carpal tunnel release in bilateral carpal tunnel syndrome. A prospective, randomised, blinded assessment. J Bone Joint Surg Br 2002;84:375-9.

15. Foucher G, Buch N, Van Overstraeten L, Gautherie M, Jesel M. [Carpal tunnel syndrome. Can it still be a controversial topic?] Chirurgie 1993;119:80-4.

16. Jacobsen MB, Rahme H. A prospective, randomized study with an independent observer comparing open carpal tunnel release with endoscopic carpal tunnel release. J Hand Surg [Br] 1996;21:202-4.

17. Macdermid JC, Richards RS, Roth JH, Ross DC, King GJ. Endoscopic versus open carpal tunnel release: A randomized trial. J Hand Surg [Am] 2003;28:475-80.

18. Saw NL, Jones S, Shepstone L, Meyer M, Chapman PG, Logan AM. Early outcome and cost-effectiveness of endoscopic versus open carpal tunnel release: A randomized prospective trial. J Hand Surg $[\mathrm{Br}]$ 2003;28:444-9. 
19. Schafer W, Sander KE, Walter A, Weitbrecht WU. [Agee endoscopic operation of carpal tunnel syndrome in comparison with open surgical technique.] Handchir Mikrochir Plast Chir 1996;28:143-6.

20. Sennwald GR, Benedetti R. The value of one-portal endoscopic carpal tunnel release: A prospective randomized study. Knee Surg Sports Traumatol Arthrosc 1995;3:113-6.

21. Stark B, Engkvist-Lofmark C. [Endoscopic operation or conventional open surgical technique in carpal tunnel syndrome: A prospective comparative study.] Handchir Mikrochir Plast Chir 1996;28:128-32.

22. Trumble TE, Diao E, Abrams RA, Gilbert-Anderson MM. Single-portal endoscopic carpal tunnel release compared with open release: A prospective, randomized trial. J Bone Joint Surg Am 2002;84-A:1107-15.

23. Palmer DH, Paulson JC, Lane-Larsen CL. Peulen VK. Olson JD. Endoscopic carpal tunnel release: A comparison of two techniques with open release. Arthroscopy 1993:9:498-508.

24. McDonough JW, Gruenloh TJ. A comparison of endoscopic and open carpal tunnel release. Wis Med J 1993;92:675-7.

25. Hallock GG, Lutz DA. Prospective comparison of minimal incision "open" and two-portal endoscopic carpal tunnel release. Plast Reconstr Surg 1995;96:941-7.

26. Worseg AP, Kuzbari R, Korak K, et al. Endoscopic carpal tunnel release using a single-portal system. Br J Plast Surg 1996;49:1-10.

27. Frick A, Baumeister RG, Kopp R. [Choice of procedure in therapy of distal median nerve compression syndrome.] Handchir Mikrochir Plast Chir 1996;28:147-50.

28. Mascharka Z. [2 1/2 years experience with endoscopic carpal tunnel release.] Handchir Mikrochir Plast Chir 1996:28:138-42.

29. Bande S, De Smet L, Fabry G. The results of carpal tunnel release: Open versus endoscopic technique. J Hand Surg [Br] 1994;19:14-7.

30. Futami T. Surgery for bilateral carpal tunnel syndrome. Endoscopic and open release compared in 10 patients. Acta Orthop Scand $1995 ; 66: 153-5$

31. Skoff HD, Sklar R. Endoscopic median nerve decompression: Early experience. Plast Reconstr Surg 1994;94:691-4.

32. Skorpik G, Landsiedl F. [Carpal tunnel syndrome. A comparison of endoscopic and open surgical treatment.] Handchir Mikrochir Plast Chir 1996;28:133-7.

33. Kerr CD, Gittins ME, Sybert DR. Endoscopic versus open carpal tunnel release: Clinical results. Arthroscopy 1994;10:266-9.

34. Bhattacharya R, Birdsall PD, Finn P, Stothard J. A randomized controlled trial of knifelight and open carpal tunnel release. J Hand Surg [Br] 2004;29:113-5.

35. Helm RH, Vaziri S. Evaluation of carpal tunnel release using the knifelight instrument. J Hand Surg [Br] 2003;28:251-4.

36. Richter VM, Bruser P. [Surgical treatment of carpal tunnel syndrome: A comparison between long and short incision and endoscopic release.] Handchir Mikrochir Plast Chir 1996;28:160-6.

37. Jugovac I, Burgic N, Micovic V, et al. Carpal tunnel release by limited palmar incision vs traditional open technique: Randomized controlled trial. Croat Med J 2002;43:33-6.

38. Mackenzie DJ, Hainer R, Wheatley MJ. Early recovery after endoscopic vs short-incision open carpal tunnel release. Ann Plast Surg 2000;44:601-4.

39. Wong KC, Hung LK, Ho PC, Wong JM. Carpal tunnel release. A prospective, randomised study of endoscopic versus limited-open methods. J Bone Joint Surg Br 2003;85:863-8.

40. Bruyns CN, Jaquet JB, Schreuders TA, Kalmijn S, Kuypers PD, Hovius SE. Predictors for return to work in patients with median and ulnar nerve injuries. J Hand Surg [Am] 2003;28:28-34.

41. Herbert R, Gerr F, Dropkin J. Clinical evaluation and management of work-related carpal tunnel syndrome. Am J Ind Med 2000;37:62-74.

42. Stock SR. Workplace ergonomic factors and the development of musculoskeletal disorders of the neck and upper limbs: A metaanalysis. Am J Ind Med 1991;19:87-107.

43. Chaise F, Bellemere P, Fril JP, Gaisne E, Poirier P, Menadi A. Returnto-work interval and surgery for carpal tunnel syndrome. Results of a prospective series of 233 patients. J Hand Surg [Br] 2004;29:568-70.

44. Provinciali L, Giattini A, Splendiani G, Logullo F. Usefulness of hand rehabilitation after carpal tunnel surgery. Muscle Nerve 2000;23:211-6 